\title{
Adaptive memory: fitness relevant stimuli show a memory advantage in a game of pelmanism
}

\author{
Stuart Wilson • Stephen Darling • Jonathan Sykes
}

Published online: 10 May 2011

(C) Psychonomic Society, Inc. 2011

In a series of articles, James Nairne, Josefa Pandeirada, and colleagues have shown that memory appears to be more efficient when information is processed in terms of fitness value (Nairne \& Pandeirada, 2008a, 2008b; Nairne, Pandeirada, Gregory, \& Van Arsdall, 2009; Nairne, Pandeirada, \& Thompson, 2008; Nairne, Thompson, \& Pandeirada, 2007). In these studies, participants were presented with a series of items that they were asked to process in a number of ways. For example, when encoding a word list, participants might be asked to process them in terms of "pleasantness." Alternatively, they might be asked to imagine that the words were "stranded in the grasslands of a foreign land" and must rate how relevant the words would be in terms of survival (see, e.g., Nairne \& Pandeirada 2008a, 2008b). The general finding is that processing in terms of survival relevance leads to superior retention when compared to processing at other levels, including levels that had previously been considered optimum for long-term retention. These findings are in line with a functional account of memory. Whilst much is understood about the processes underlying memory-for example, the benefits of using visual imagery in memory tasks (Quinn \& McConnell, 1996a, 1996b) or level-ofprocessing effects (Craik \& Lockhart, 1972)—only recently have researchers addressed questions relating to why nature

\footnotetext{
S. Wilson $(\bowtie) \cdot$ S. Darling

Psychology \& Sociology, School of Arts, Social Sciences and Management, Queen Margaret University,

Queen Margaret University Drive, Musselburgh,

East Lothian EH21 6UU Scotland, UK

e-mail: swilson@qmu.ac.uk

J. Sykes

School of Engineering and Computing,

Glasgow Caledonian University,

Glasgow, Scotland, UK
}

has provided us with a memory system structured as it is. Naturally, such considerations lend themselves to evolutionary accounts, which would lead to the suggestion that the memory phenomena that we observe are a reflection of the selection pressures that were present during our evolutionary history. Although identifying cognitive adaptations is notoriously difficult, it is possible to hypothesise within an evolutionary framework (see, e.g., Klein, Cosmides, Tooby, \& Chance, 2002). Accordingly, a growing number of researchers are approaching memory in this way (see Kang, McDermott, \& Cohen, 2008; Otgaar, Smeets, \& van Bergen, 2010; Weinstein, Bugg, \& Roediger, 2008), and the results seem to be consistent with the notion that memory does, at least to some degree, differentially deal with information that is processed according to a survival scenario, as compared to when information is processed in other ways.

Nairne and his colleagues have so far resisted using stimuli that are likely to be inherently fitness relevant, instead focussing on how neutral stimuli are remembered under a variety of contexts, arguing that the survival relevance of a stimulus is context dependent (Nairne et al., 2007). Although this argument is entirely valid, it is unlikely to be equally true for all classes of stimuli. Instead, some stimuli are likely to be privileged in terms of the way our cognitive systems deal with them, as a result of their fitness relevance. As Nairne et al. (2007) pointed out, not all environmental stimuli are created equal. If natural selection has shaped memory systems, it would be unsurprising if certain stimuli were afforded special status, given their importance for survival and their pervasive presence in the environment in which we (and our mammalian ancestors) evolved. We might therefore expect that stimuli resembling predators or other threats to ancestral survival will be more keenly detected and remembered. Öhman and Mineka (2001, 2003) have 
demonstrated that objects such as snakes might be considered "special" stimuli and posited an evolved module for fear learning and elicitation that is activated by such evolutionarily relevant stimuli. Similarly, Gerdes, Uhl, and Alpers (2009) tentatively suggested that spiders are another special class of stimuli, having found them to be classed by participants as being more fear inducing, more disgusting, and more dangerous than other potentially dangerous arthropods (e.g., bees/wasps). Stimuli such as snakes represent the oldest threats to mammals (see, e.g., Isbell, 2006). Primates would also have had experienced threats from attacking felines from around 50 million years ago (Öhman, 2007), and more generally should have evolved to be sensitive to indications relating to predation threats, particularly animals displaying aggressive or threatening signals (Barrett, 2005). Additionally, as a result of being a "generalist" species with regards to food consumption, humans also appear to have an acute "contagion avoidance" response that is highly sensitive and triggers strong disgust emotions (see, e.g., Rozin, Millman, \& Nemeroff, 1986). This would suggest that potential contaminators may also be afforded special status.

There is some evidence to support the suggestion that evolutionarily relevant stimuli are attended to more efficiently than other stimuli, although there is a lack of consensus. New, Cosmides, and Tooby (2007) used a change detection paradigm to argue that humans were better at detecting changes involving animals rather than inanimate objects, a finding that they suggested reflects ancestral priorities. LoBue and DeLoache (2008) found that both children (3-5 years old) and adults were more efficient at detecting snakes in a visual array than at detecting other, nonthreatening stimuli (flowers, frogs, and caterpillars), which LoBue and DeLoache suggested was consistent with Öhman and Mineka's (2001) "fear module."

Other authors, such as Brosch and Sharma (2005), Blanchette (2006), and Fox, Griggs, and Mouchlianitis (2007), have found that adaptively relevant stimuli are not always attended to more efficiently than survival-relevant modern stimuli, arguing that "fear relevance" is the important factor in determining attention, rather than the evolutionary relevance of the stimulus itself. However, it is important to note that the outcomes of these studies may have been biased by the selection of the stimuli. Brosch and Sharma used images (modern threatening, ancestral threatening, and neutral) obtained via the Internet and did not attempt to match the two classes of threatening stimuli on any other dimension. Blanchette did not indicate the source of her stimuli and seems not to have matched the evolutionarily relevant fear stimuli and the modern fearrelevant stimuli on any other factors. Fox et al. used stimuli from the International Affective Picture System (IAPS; Lang, Bradley, \& Cuthbert, 2005) but did not match fearrelevant stimuli on any measure, instead relying on a pilot study in which pictures were rated for fear relevance. Given that fear-relevant pictures in the IAPS are often associated with different arousal ratings, it is possible that this study is not a fair comparison between phylogenetic and ontogenetic fear-relevant stimuli, a criticism that could also apply both to Brosch and Sharma and to Blanchette.

The purpose of the present research was to test a hypothesis relating to the way our memories deal with specific types of stimuli. If memory is an adaptation, we would expect to find the most striking effects when it is employed to process stimuli that are similar to those that ancestors (human and mammalian) would have frequently encountered and that had clear immediate survival relevance. To test this, we used a computer version of the game commonly known as "match" (a.k.a. pelmanism, concentration, memory, pairs, or pexeso). Our primary hypothesis was that participants would be more efficient at matching pairs of stimuli that were directly related to fitness than at matching other pairs of stimuli. We also predicted that, on trials on which the wrong target was selected, participants would be closer to the correct target when they were looking for a fitness-relevant stimulus as compared to when they were looking to match other stimuli. The reason for the secondary hypothesis was as follows: If memory did evolve in order to be survival enhancing, it might not always be as precise as we would like it to be in the laboratory. Instead, it might be the case that, when we are presented with a survival-relevant stimulus in our environment, we remember the general location (e.g., "snake in this general area") rather than its specific location.

\section{Method}

Participants

A total of 99 participants took part, all of whom were Queen Margaret University students. Some of these students took part for course credit, whilst others took part in response to e-mail requests and word of mouth. Participants' ages ranged from 18 to $62(M=24.10, S D=7.98)$. All participants gave informed consent, and local ethical approval was obtained prior to testing.

\section{Materials}

A match game was created, consisting of $5 \mathrm{x} 4$ grids of stimuli, using Unity 3D software. The grids were populated with pairs of identical images, with five pairs of target and five pairs of filler stimuli. The target stimuli were survival-relevant items (i.e., potentially life threatening), whereas fillers were not. All images were obtained from the International Affective Picture System (Lang et al., 2008). The IAPS is a large set of well- 
validated pictures that have been individually assigned three ratings; valence, arousal, and dominance. For the purposes of the present study, valence and arousal were the dimensions on which stimuli were chosen. Two grids were used, one of which contained evolutionarily relevant survival-related stimuli, the other of which contained evolutionarily irrelevant survival-related stimuli. Materials for the evolutionarily relevant grid consisted of five images of stimuli likely to have been encountered frequently during human evolution and that have clear immediate survival relevance (mean valence/arousal $=3.7 / 6.3$ ). Most of these stimuli represented predators or dangerous animals, and one represented a contagion threat (cockroaches on food). For the evolutionarily irrelevant grid, the five images comprised items that were unlikely to have been encountered in human evolutionary history, despite having clear immediate survival relevance (mean valence/arousal $=2.7 / 6.4)$. Examples included a gun aimed directly toward the observer and a knife being held in a "stabbing" position. Both sets of target stimuli were roughly matched on valence (in that all images were towards the lower end of the scale) and, more importantly, were matched on arousal. Arousal was given priority during the selection process because it was felt that any memory effects that might be found should not be a function of how arousing a particular set of stimuli were (see Cavenett \& Dixon, 2006, for a study of how arousal can influence memory for emotionally relevant stimuli, whilst valence has no effect). To this effect, the two sets of survival-relevant stimuli did not significantly differ from each other on arousal (Mann-Whitney $U=11.5$, $p=.83$ ). In each grid, five pairs of filler stimuli were also included. These filler stimuli would not be expected to have any significant immediate survival relevance in either an evolutionarily historical or a modern-day context. Two sets of five filler items were used in the grids: one set of nonthreatening animals and another of everyday objects (which also included one image of an elderly man facing away from the observer, looking out of a window). The animal and object filler sets did not differ significantly on arousal (Mann-Whitney $U=8, p=.35$ ). ${ }^{1}$

\section{Design and procedure}

The participants took part in two experimental "match" games. In each game, participants viewed a $5 \times 4$ array of squares. They were asked to click on a square (which resulted in a stimulus image being revealed) and then to attempt to find a match for that image by clicking on another square in the grid. Once a second square had been selected, the two selected

\footnotetext{
${ }^{1}$ The library numbers for the IAPS pictures (Lang et al., 2005) used in this study are $1120,1200,1300,1602,1610,1620,1670,1910,1726$, $2480,6213,6260,6300,7006,7080,7130,7380,9600,9622$, and 9635.2 .
}

images remained visible in their grid locations for approximately $2 \mathrm{~s}$, after which time the squares were either removed from the game (on correct-match trials) or the stimulus images were hidden again (on incorrect-match trials). Participants continued this process until they had successfully matched all pairs. Participants first completed a practice grid in which the stimuli consisted of pairs of geometric shapes before completing the two experimental games. In both games, the stimuli were situated randomly within the grid by the program at the beginning of the session. In one game, the target pairs consisted of evolutionarily relevant stimuli, whilst in the other, the target pairs were evolutionarily irrelevant stimuli. Filler type was counterbalanced across participants, so half of the grids with evolutionarily relevant targets contained animal fillers, and half contained object fillers. The same counterbalancing was applied to grids with evolutionarily irrelevant targets. Participants who had seen animals with the evolutionary targets saw objects with the nonevolutionary targets, and vice versa (Fig. 1).

The primary focus was whether participants would perform better at remembering the evolutionarily relevant targets. A turn represented the turning over of a pair of stimuli. The primary dependent variable was the total number of errors (per participant and condition) made on target trials. The secondary dependent variable was "error distance," which represents the total distance (per condition and participant) in screen pixels from the centre of all incorrectly chosen items to the centre of their respective correct items; the latter measure is an estimate of overall inaccuracy in spatial memory on trials on which the correct item was not recalled.

\section{Results}

The data from 3 participants were excluded on the basis that these participants were extreme in terms of the number of turns taken to match the target stimuli in either condition; extreme values were defined as those that were more than three standard deviations in excess of the mean. A further 9 participants were excluded due to a data recording error.

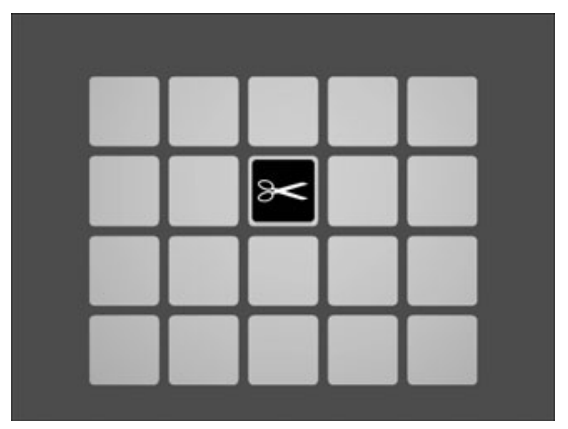

Fig. 1 Black-and-white screen shot of stimulus array in the practice condition (stimuli in the experimental conditions were colour) 
The number of correct responses was invariant, because items were removed from the grid if answered correctly. Hence, the analysis focuses on errors. Table 1 shows the mean numbers of errors of different types made by participants. The total number of errors of all types made on target trials was smaller for evolutionarily relevant targets $(M=4.31, S D=$ 1.76) than for evolutionarily irrelevant targets $(M=4.71, S D=$ 2.01) $[t(85)=1.66, p=.05$ (one-tailed), $d z=0.18]$.

When participants misremembered the correct location of the paired item, the error distance in screen pixels was significantly smaller on evolutionarily relevant trials $(M=$ $1,367.15, S D=620.83)$ than on evolutionarily irrelevant trials $(M=1,539.24, S D=707.03)[t(85)=2.02, p=.02$ (one-tailed), $d z=0.22]^{2}$

There was no evidence of differential responding across filler items, either in terms of number of errors (animals, $M=4.37, S D=2.45$; objects, $M=4.45, S D=2.10$ ) $[t(85)=.24, p=.40$ (one-tailed), $d z=0.03]$ or error distance (animals, $M=1,445.54, S D=847.11$; objects, $M=1,430.33$, $S D=734.20)[t(85)=0.24, p=.45$ (1-tailed), $d z=.01]$.

\section{Discussion}

It is becoming increasingly clear that evolutionary imperatives have shaped human memory. It is not controversial to posit that our memory capacities served some form of adaptive function and were shaped by our evolutionary history, although the extent to which these features persist in our current cognitive machinery is less clear. If we consider memory as adaptive, its purpose is likely to be to help organisms coordinate current and future behaviour in line with previous experience. If certain stimuli were consistently encountered over the course of our evolutionary history, and if these stimuli had consequences for survival, it would be unsurprising if we were to find that certain memory functions were especially fine-tuned in dealing with such items. Furthermore, the form that such effects may take is likely to be related to spatial memory, because remembering the location of survival-threatening objects will facilitate their avoidance. Our results suggest that this may be the case, suggesting that some mnemonic function is rooted in our evolution, rather than in either developmental factors or current selection pressures.

\footnotetext{
${ }^{2}$ It is, of course, the case that total error distance per participant increases with the total number of errors. However, we carried out an analysis using the average error distance per move (for each participant and condition) as the dependent variable. This averaged error distance was still significantly lower in the evolutionarily relevant $(M=141.30, S D=42.58)$ than in the evolutionarily irrelevant $(M=152.02, S D=43.76)[t(85)=1.86, p$ (one-tailed $)=.03, d z=.20]$ condition.
}

Table 1 Mean numbers of error responses to the different stimulus types

\begin{tabular}{lll}
\hline Response type & \multicolumn{2}{l}{ To-Be-Remembered Stimulus Type } \\
\cline { 2 - 3 } & $\begin{array}{l}\text { Evolutionarily } \\
\text { relevant }\end{array}$ & $\begin{array}{l}\text { Evolutionarily } \\
\text { irrelevant }\end{array}$ \\
\hline Selection of an alternative target & $1.83(1.02)$ & $2.13(1.53)$ \\
Selection of a filler & $2.49(1.49)$ & $2.58(1.59)$ \\
\hline
\end{tabular}

Standard deviations are in parentheses.

This conclusion is broadly in line with recent work on adaptive memory, most notably in the work of Nairne and Pandeirada (e.g., Nairne \& Pandeirada, 2008a, b; Nairne et al., 2009). However, whilst they emphasised the advantage of processing general information in survival contexts, the present work focuses more on the way our memories are tuned to specific classes of stimuli. Öhman and Mineka (2001, 2003) have postulated that a fear module exists. One property that these authors have suggested that such a module would have is that it would be activated by specific stimuli that are fear relevant from an evolutionary perspective (Öhman \& Mineka, 2001, p. 483). It follows that such a module would require a degree of dedicated memory function relating to the relevant stimuli. Our work suggests that evolutionarily pertinent survival-related items are remembered better; therefore, we tentatively speculate that this fear module is linked to memory, although the exact form that such a link would take is a question for future empirical work.

The present study represents a first step, and caution must be exercised against drawing strong conclusions on the basis of a single study. Much work remains to be done concerning the nature of the stimuli that may (or may not) receive priority in memory processing. One potential issue is that the "modern" threat stimuli used in this study incidentally included some people. It is possible to argue that other humans posed a significant threat throughout human evolution, and thus that our evolutionarily irrelevant stimuli could not be considered truly "modern." However, we were careful in selecting the images that were used as "modern" survivalrelevant stimuli and selected those in which the threat was primarily (and sometimes solely) from the modern weapon being brandished. None of the images showed humans with "aggressive" facial expressions. Indeed, in two of the three images involving humans with weapons, the only part of the human that was visible was the hand that the weapon was in. Consequently, the arousal levels, as recorded in the IAPS data, were likely to be a function of the weapon. Using pictures of weapons in isolation was not an option, because the arousal figures for these images were different from those for the evolutionarily relevant survival stimuli. On a related note, humans represent an ambiguous stimulus that may occasionally be threatening, but usually is not. Even an aggressive 
human is not clearly a direct threat, because (s)he may be being aggressive for your (or your group's) benefit. Encountering a dangerous animal, on the other hand, is almost always likely to be processed as a threat in the first instance. For these reasons, we believe the comparison between the "old" and "modern" threats to be a valid one. Furthermore, the fact that a difference in memory performance was identified between the evolutionarily relevant and irrelevant stimuli suggests that there is a difference of some kind between these stimuli, and we argue that evolutionary relevance (the aspect we set out to manipulate) is the most likely source of this difference. This is not to say that there are no further questions to be addressed concerning the stimuli. Further work needs to be done, for example, on holding valence constant and on probing the fear and threat levels that participants attach to the stimuli. Additionally, further investigation will be required in order to determine the extent to which the stimuli we characterised as "evolutionarily relevant" are similar. Is a snake remembered any differently from an attacking feline, for example?

In addition to questions surrounding the stimuli, there are further issues relating to the memory systems implicated. What, for example, might be the role of episodic memory in providing organisms with survival-relevant data that can inform current actions? Can an effect similar to the one described be established in other memory tasks? The present study used a familiar game in order to test the hypothesis in a novel way, but it remains to be seen whether such effects can be elicited using other techniques.

In conclusion, our data serve as a first step in establishing that certain items receive preferential processing by our memory systems in line with our evolutionary history. We present these data in the hope that other researchers will use the paradigm as a means of answering the questions raised by our findings. Much work remains to be done to further our understanding of such effects, but the present research may serve as a jumping-off point for a new research direction looking at the relationship between evolution and the structures and contents of memory.

Author Note Thanks are due to Chris Brough and Lynne Newlands for help with data collection and recruitment, and Craig Stevenson for writing the program. Thanks also to Queen Margaret University and Glasgow Caledonian University for supporting this research, and to three anonymous reviewers for their useful comments and suggestions.

\section{References}

Barrett, H. C. (2005). Adaptations to predators and prey. In D. Buss (Ed.), The handbook of evolutionary psychology (pp. 200-223). Hoboken: John Wiley \& Sons.

Blanchette, I. (2006). Snakes, spiders, guns, and syringes: How specific are evolutionary constraints on the detection of threatening stimuli? Quarterly Journal of Experimental Psychology, $59,1484-1504$.
Brosch, T., \& Sharma, D. (2005). The role of fear-relevant stimuli in visual search: A comparison of phylogenetic and ontogenetic stimuli. Emotion, 5, 360-364.

Cavenett, T., \& Dixon, R. D. V. (2006). The effect of arousal on memory for emotionally-relevant information: A study of skydivers. Behavior Research and Therapy, 44, 1461-1469.

Craik, F. I. M., \& Lockhart, R. S. (1972). Levels of processing: A framework for memory research. Journal of Verbal Learning and Verbal Behavior, 11, 671-684.

Fox, E., Griggs, L., \& Mouchlianitis, E. (2007). The detection of fearrelevant stimuli: Are guns noticed as quickly as snakes? Emotion, 7, 691-696.

Gerdes, A. B. M., Uhl, G., \& Alpers, G. W. (2009). Spiders are special: Fear and disgust evoked by pictures of arthropods. Evolution and Human Behavior, 30, 66-73.

Isbell, L. A. (2006). Snakes as agents of evolutionary change in primate brains. Journal of Human Evolution, 51, 1-35.

Kang, S. H. K., McDermott, K. B., \& Cohen, S. M. (2008). The mnemonic advantage of processing fitness-relevant information. Memory \& Cognition, 36, 1151-1156.

Klein, S. B., Cosmides, L., Tooby, J., \& Chance, S. (2002). Decisions and the evolution of memory: Multiple systems, multiple functions. Psychological Review, 109, 306-329.

Lang, P. J., Bradley, M. M., \& Cuthbert, B. N. (2005). International affective picture system (IAPS): Affective ratings of pictures and instruction manual (Tech. Rep. A-6). Gainesville: University of Florida.

Lang, P. J., Bradley, M. M., \& Cuthbert, B. N. (2008). International affective picture system (IAPS): Affective ratings of pictures and instruction manual (Tech. Rep. A-8). Gainesville: University of Florida.

LoBue, V., \& DeLoache, J. S. (2008). Detecting the snake in the grass: Attention to fear-relevant stimuli by adults and young children. Psychological Science, 19, 284-289.

Nairne, J. S., \& Pandeirada, J. N. S. (2008a). Adaptive memory: Is survival processing special? Journal of Memory and Language, 59, 377-385.

Nairne, J. S., \& Pandeirada, J. N. S. (2008b). Adaptive memory: Remembering with a stone-age brain. Current Directions in Psychological Science, 17, 239-243.

Nairne, J. S., Pandeirada, J. N. S., Gregory, K. J., \& Van Arsdall, J. E. (2009). Adaptive memory: Fitness relevance and the hunter gatherer mind. Psychological Science, 20, 740-746.

Nairne, J. S., Pandeirada, J. N. S., \& Thompson, S. R. (2008). Adaptive memory: The comparative value of survival processing. Psychological Science, 19, 176-180.

Nairne, J. S., Thompson, S. R., \& Pandeirada, J. N. S. (2007). Adaptive memory: Survival processing enhances retention. Journal of Experimental Psychology: Learning, Memory, and Cognition, 33, 263-273.

New, J., Cosmides, L., \& Tooby, J. (2007). Category-specific attention for animals reflects ancestral priorities, not expertise. Proceedings of the National Academy of Sciences, 104, 16593-16603.

Öhman, A. (2007). Has evolution primed humans to "beware the beast"? Proceedings of the National Academy of Sciences, 104, 16396-16397.

Öhman, A., \& Mineka, S. (2001). Fear, phobias and preparedness: Toward an evolved module of fear and fear learning. Psychological Review, 108, 483-522.

Öhman, A., \& Mineka, S. (2003). The malicious serpent: Snakes as a prototypical stimulus for an evolved module of fear. Current Directions in Psychological Science, 12, 5-9.

Otgaar, H., Smeets, T., \& van Bergen, S. (2010). Picturing survival memories: Enhanced memory after fitness-relevant processing occurs for verbal and visual stimuli. Memory \& Cognition, 38, $23-28$. 
Quinn, J. G., \& McConnell, J. (1996a). Indications of the functional distinctions between the components of visual working memory. Psychologische Beiträge, 38, 355-367.

Quinn, J. G., \& McConnell, J. (1996b). Irrelevant pictures in visual working memory. Quarterly Journal of Experimental Psychology, $49 A, 200-215$.
Rozin, P., Millman, L., \& Nemeroff, C. (1986). Operation of the laws of sympathetic magic in disgust and other domains. Journal of Personality and Social Psychology, 50, 703-712.

Weinstein, Y., Bugg, J. M., \& Roediger, H. L., III. (2008). Can the survival recall advantage be explained by basic memory processes. Memory \& Cognition, 36, 913-919. 